\title{
Low Vitamin D Level was Associated with Non-alcoholic Fatty Liver Disease in Patients with Type 2 Diabetes Mellitus
}

\author{
Vahid Sheikhi (iD ${ }^{1}$ and Zahra Heidari (iD) ${ }^{2,}$ \\ ${ }^{1}$ Department of Pediatric Nephrology, Zahedan University of Medical Sciences, Zahedan, Iran \\ ${ }^{2}$ Department of Endocrinology and Metabolism, Zahedan University of Medical Sciences, Zahedan, Iran \\ "Corresponding author: Department of Endocrinology and Metabolism, Zahedan University of Medical Sciences, Zahedan, Iran. Email: z.heidari10@yahoo.com \\ Received 2021 July 22; Revised 2021 September 29; Accepted 2021 October 01.
}

\begin{abstract}
Background: Non-alcoholic fatty liver disease (NAFLD) and type 2 diabetes mellitus (T2DM) are major public health concerns. Besides the known risk factors, other risk factors, such as vitamin D deficiency, have been suggested for NAFLD.

Objectives: This cross-sectional research aimed to investigate the relationship between serum vitamin D levels and NAFLD in a group of patients with T2DM.

Methods: We investigated various clinical and biochemical parameters, including serum vitamin D level, liver function tests, and liver sonography in 1,110 adult patients with T2DM. The mean difference of numerical variables in NAFLD and non-NAFLD groups was analyzed with an independent sample $t$-test. Chi-square test was used to evaluate the association between two categorical variables. Results: Out of 1,110 patients with T2DM, 837 (75.4\%) had NAFLD. The mean vitamin D level in diabetic patients with NAFLD was significantly lower than non-NAFLD group ( $19.71 \mathrm{ng} / \mathrm{mL}$ vs. $27.68 \mathrm{ng} / \mathrm{mL}$, respectively; P < 0.001). Furthermore, 410 (49\%) patients with NAFLD were found with vitamin D deficiency, while this value was 85 (31.1\%) in non-NAFLD group. According to the results of univariate logistic regression analysis, vitamin $D$ deficiency $(O R=3.87)$ and insufficient vitamin $D(O R=2.83)$ were the significant variables for NAFLD.
\end{abstract}

Conclusions: There was a significant association between vitamin D deficiency and NAFLD in patients with T2DM.

Keywords: Type 2 Diabetes Mellitus, Non-alcoholic Fatty Liver Disease, Vitamin D

\section{Background}

Non-alcoholic fatty liver disease (NAFLD) is defined as the aggregation of triglycerides within hepatocytes exceeding $5 \%$ of liver weight. It is not caused by excessive alcohol use or different steatosis sources (1). It encompasses a wide spectrum of liver pathologies, with NAFLD at one end of the spectrum followed by non-alcoholic steatohepatitis (NASH), liver cirrhosis, and hepatocellular carcinoma at the other end (2). In some patients, NAFLD progresses to end-stage liver disease, which has made NAFLD a major reason for morbidity and mortality over the last two decades; it is predicted that NAFLD will be the number one etiology of liver transplantation worldwide (3). Evidence suggests that advanced liver fibrosis can be caused by mild degrees of steatosis and inflammation of the liver (4). Today, many experts believe that a lot of patients diagnosed with cryptogenic cirrhosis have NAFLD/NASH as an underlying disease (5).

NAFLD and type 2 diabetes mellitus (T2DM) have been known as major public health concerns. The prevalence of NAFLD in Western countries is $46.2 \%$, and it is the most com- mon liver disease. Its prevalence in some specific groups, such as obese people and patients with T2DM, reaches 75 to $90 \%$. In recent decades, the prevalence of NAFLD has increased along with obesity worldwide, reaching $46.2 \%$ in Europe, 33\% in North America, and 31.8\% in Asia. It is estimated that about one-fourth of the world's population suffers from NAFLD. Also, the global prevalence of diabetes in 2019 was $9.3 \%$ (463 million people), which is expected to increase to $10.2 \%$ (578 million) by 2030 and $10.9 \%$ (700 million) by 2045. Notably, patients with NAFLD experience T2DM and vice versa. About 25\% of patients with NAFLD and $50 \%$ of patients with NASH have T2DM, while NAFLD is reported in about $70 \%$ of patients with T2DM. These two conditions have mutual effects on each other (6-11). In patients with T2DM, NAFLD increases the risk of mortality. Also, the presence of T2DM causes a three-fold increase in the risk of progressive liver fibrosis and a two-fold increase in the risk of hepatocellular carcinoma. It is also an independent predictor of liver disease mortality and all-cause mortality (12).

Insulin resistance is a common hallmark of NAFLD and 
T2DM, and NAFLD is a hepatic component of metabolic syndrome (13). Metabolic diseases, such as hypertension, visceral obesity, and dyslipidemia are known risk factors for NAFLD (14). Besides the known risk factors, other risk factors, such as vitamin D deficiency, have been recently suggested for NAFLD. Previous research shows that vitamin D deficiency can enhance the risk of insulin resistance and metabolic syndrome (15). Vitamin D is involved in NAFLD by exerting anti-inflammatory and anti-fibrotic effects on liver cells. It exerts these effects through inflammatory cytokines, like tumor necrosis factor- $\alpha$ (TNF- $\alpha$ ), interleukin6 (IL-6), and IL-1 $\beta$, as well as adipokines, like leptin and adiponectin (16). In addition, it has been shown that vitamin D can decrease the cytokeratin 18 apoptotic fragment M30 concentration as an indicator for liver damage (17). In patients with T2DM, vitamin D deficiency can reduce the expression of glucose transporters on the cell surface, reduce glucose export from the liver, and stimulate intrahepatic lipid synthesis, thereby contributing to the pathogenesis of NAFLD in these patients (18).

Low serum vitamin D levels are associated with NAFLD and are involved in its pathogenesis (19-22). However, this association has not been confirmed in all previous studies, and no relationship has been suggested between the serum vitamin D level and NAFLD in some studies $(23,24)$.

\section{Objectives}

This cross-sectional research aimed to investigate the relationship between serum vitamin D levels and NAFLD in a group of patients with T2DM.

\section{Methods}

The current cross-sectional research was performed on 1,110 patients with T2DM (age range: 31 - 75years) referring to endocrine clinics of Zahedan, Iran, from March 2018 to August 2020. T2DM was diagnosed based on American Diabetes Association (ADA) criteria (24). A physician completed an information form, including the patient's age, sex, duration of diabetes, co-morbidities, smoking, alcohol consumption, and drug history.

Participants with evidence of any chronic liver disease, such as autoimmune hepatitis, hemochromatosis, viral hepatitis, primary biliary cirrhosis, Wilson's disease, and any evidence of liver cirrhosis were excluded from the study. Also, people with other types of diabetes, such as gestational DM, latent autoimmune diabetes of adults, and type $1 \mathrm{DM}$, were excluded from the study. We also excluded all individuals with acute infection, decreased renal function $\left(\mathrm{eGFR}<60 \mathrm{ml} / \mathrm{min} / 1.73 \mathrm{~m}^{2}\right.$ or plasma creatinine $>2$ $\mathrm{mg} / \mathrm{dL}$ ), malignancy, thyroid dysfunction, or alcohol consumption of any volume. Patients with a history of taking any supplements, including vitamin $\mathrm{D}$, as well as pregnant and lactating women were also excluded from the study.

The patients' height, weight, and blood pressure were evaluated. The weight was measured with minimal clothing by a digital scale, and height was measured while standing without shoes by a stadiometer. Body mass index (BMI) was determined using this formula: weight (kg) divided by height $\left(\mathrm{m}^{2}\right)$. Patients' blood pressure was measured after 15 minutes of rest and before blood sampling using a manual sphygmomanometer.

For all subjects, liver ultrasonography was performed by a sonologist after 12 hours of fasting. Fatty liver was determined based on standard criteria, including liver illumination, variation between the liver and kidneys echogenicity, and the degree of ambiguity of blood vessels. Grading of fatty liver based on the amount of fat deposition in the liver was determined as follows: (1) Grade I - observable periportal and diaphragmatic echogenicity in association with increased liver echogenicity; (2) Grade II - non-observable periportal echogenicity in association with increased liver echogenicity without diaphragmatic ambiguity; and (3) Grade III - non-observable periportal echogenicity in association with increased liver echogenicity with diaphragmatic ambiguity (25). NAFLD was diagnosed according to the American Gastroenterological Association criteria as follows: (1) presence of hepatic steatosis on imaging or histology; (2) no excessive use of alcohol; (3) no other reasons for hepatic steatosis, and (4) no other synchronic reason for chronic liver disease (26).

Fasting venous blood was collected for measurement of the glycemic profile, thyroid function tests, and other biochemical tests. Blood sampling was done between 8 , and 9 am following 12 hours of fasting. Plasma glucose was measured with the glucose oxidase method. Measurement of glycated hemoglobin (HbA1c) was carried out using high-performance liquid chromatography (HPLC). Lipids were measured using enzymatic colorimetric tests. Blood urea nitrogen (BUN), creatinine, and liver function tests were assessed by enzymatic colorimetric assays. Serological tests for hepatitis $\mathrm{B}$ and $\mathrm{C}$ rejection were performed in patients with elevated liver enzymes. The normal AST and ALT were defined as less than $40 \mathrm{u} / \mathrm{L}$. The 25hydroxyvitamin $\mathrm{D}(25(\mathrm{OH}) \mathrm{D})$ test was performed using the enzyme immunoassay method. Values less than $20 \mathrm{ng} / \mathrm{mL}$ were considered as vitamin D deficiency and values of 20 to $30 \mathrm{ng} / \mathrm{mL}$ were regarded as insufficiency (27).

All experiments were performed according to the ethical standards of the institutional and/or national research committee and the 1964 Helsinki declaration and its later amendments. The study protocol was approved by the Ethics Committee for Human Studies at Zahedan University of Medical Sciences. Informed consent was obtained from all participants. 


\subsection{Statistical Analysis}

Continuous and categorical data are presented as mean \pm standard deviation (SD) and frequency (percentage), respectively. Also, we presented the data with histogram and box plot, as appropriate. The normality of variables was assessed with Shapiro-Wilk test and graphical approaches. The mean difference of numerical variable in NAFLD and non-NAFLD groups was analyzed using the independent sample $t$-test. Chi-square test was used to evaluate the association between two categorical variables. The association between independent factors and NAFLD was assessed using univariate and multivariate logistic regression models. The multivariate logistic regression model was conducted based on the backward stepwise method. A P-value less than 0.05 was considered a significant difference. Data analysis was conducted using Stata software (Release 14. College Station, TX: StataCorp LP).

\section{Results}

In this study, out of 1,110 patients with T2DM, 837 (75.4\%) subjects had NAFLD. Among the patients, 64.9\% were females, and gender distribution was not significantly different in patients with and without NAFLD $(\mathrm{P}=0.375)$. The mean age in NAFLD group was significantly higher than non-NAFLD group (54.12 vs. 49.41 years, respectively; $\mathrm{P}<$ 0.001). The mean duration of diabetes in patients with NAFLD (10.63 years) was almost twice that of patients without NAFLD (5.84 years), indicating a statistically significant difference $(\mathrm{P}<0.001)$. A comparison of other clinical and laboratory characteristics between the two groups is shown in Table 1.

The mean vitamin D level in NAFLD group was significantly lower than non-NAFLD group (19.71 ng/mL vs. $27.68 \mathrm{ng} / \mathrm{mL}$, respectively; $\mathrm{P}<0.001)$. According to the 25-OH vitamin D status classification, 410 (49\%) patients with NAFLD had vitamin D deficiency, while this value was 85(31.1\%) for patients without NAFLD (Figure 1). There was no statistically significant difference in the blood sampling seasonal distribution between the two groups ( $\mathrm{P}=$ 0.934).

In univariate logistic regression analysis, HbA1c with an odds ratio (OR) of 8.51 and history of insulin use (OR = 5.35) showed the highest OR for NAFLD. Also, duration of diabetes $(\mathrm{OR}=2.24)$, family history of diabetes $(\mathrm{OR}=2.84)$, history of taking the antihypertensive drug $(\mathrm{OR}=2.15)$, vitamin $\mathrm{D}$ deficiency compared to normal vitamin $\mathrm{D}(\mathrm{OR}=$ 3.87), and insufficient vitamin D compared with normal vitamin $\mathrm{D}(\mathrm{OR}=2.83)$ were the significant variables with $\mathrm{OR}$ $>2$ for NAFLD (Table 2 ).

According to the multivariate logistic model, after eliminating the confounding effect of other variables, the chance of developing NAFLD in patients with vitamin D deficiency was 3.15 times higher than patients with normal vitamin D levels. In the multivariate model, the history of insulin consumption ( $\mathrm{OR}=20.3)$, HbA1c $(\mathrm{OR}=11.76)$, and the duration of diabetes $(\mathrm{OR}=2.92)$ were the most important variables for NAFLD (Table 3 ).

\section{Discussion}

According to our results, the serum vitamin D level was lower in diabetic patients with NAFLD compared to those in non-NAFLD group. Also, vitamin D deficiency was associated with NAFLD in these patients.

These findings are consistent with some previous studies, indicating that the serum vitamin D level was lower in diabetic cases with NAFLD $(28,29)$. In this regard, a study by Rhee et al. showed that the serum vitamin D levels were lower in NAFLD patients compared with the control group (30). Another study showed that the serum vitamin D levels were significantly lower in patients diagnosed with NAFLD considering liver biopsy than in the control group (31). However, some studies have reported different results regarding the relationship between vitamin D levels and NAFLD. Two different studies carried out in China (32) and Korea (33) reported no significant difference between patients with and without NAFLD regarding the serum vitamin D level.

The discrepancy between the results of different studies can be attributed to factors such as different methods and designs, different criteria for NAFLD diagnosis, different definitions for vitamin D deficiency, lack of matched study groups for interfering factors (such as BMI), and selection bias in cross-sectional studies. Also, genetic factors, such as polymorphisms in vitamin D receptor genes, may be involved. Therefore, vitamin D may affect the evolution and advancement of NAFLD only in subjects with specific genotypes (34).

Previous studies have shown that vitamin D considerably affects immune system regulation, cell differentiation, regulation of cell proliferation, and inflammatory processes. Vitamin D can improve insulin secretion and reduce insulin resistance and liver fibrosis. Through these mechanisms, which are mediated by cytokines and adipokines, vitamin D may contribute to the evolution and advancement of NAFLD (35). Numerous studies have shown that markers of inflammation, such as CRP, TNF$\alpha$, and IL- 6 are possibly associated with the pathogenesis of NAFLD (36). Elevated serum TNF- $\alpha$ levels have been associated with the increased risk of NAFLD in healthy nondiabetic individuals (36). Moreover, a direct relationship has been found between the increased serum levels of inflammatory markers and NAFLD severity (37). Overall, vitamin D can reduce inflammation in various ways (38). Therefore, it can be proposed that vitamin $\mathrm{D}$ reduces the 


\begin{tabular}{|c|c|c|c|c|}
\hline \multirow{2}{*}{ Variables } & \multirow{2}{*}{$\operatorname{All}(\mathbf{n}=1110)$} & \multicolumn{2}{|c|}{ NAFLD Status } & \multirow{2}{*}{ P-Value } \\
\hline & & $\operatorname{NAFLD}(\mathbf{n}=\mathbf{8 3 7})$ & Non-NAFLD $(\mathbf{n}=273)$ & \\
\hline Age (y) & $52.96 \pm 10.46$ & $54.12 \pm 11.22$ & $49.41 \pm 6.54$ & $<0.001$ \\
\hline Sex, female & $720(64.9)$ & $549(65.6)$ & $171(62.6)$ & 0.375 \\
\hline Diabetes duration (y) & $9.45 \pm 4.24$ & $10.63 \pm 4.15$ & $5.84 \pm 1.66$ & $<0.001$ \\
\hline Positive family history of DM & $780(70.3)$ & $636(76.0)$ & $144(52.7)$ & $<0.001$ \\
\hline Use of antihypertensive drug & $601(54.1)$ & $492(58.8)$ & $109(39.9)$ & $<0.001$ \\
\hline Use of statin & $939(84.6)$ & $711(84.9)$ & $228(83.5)$ & 0.570 \\
\hline Use of OHA & $644(58.0)$ & $449(53.6)$ & $195(71.4)$ & $<0.001$ \\
\hline Use of insulin & $364(32.8)$ & $334(39.9)$ & $30(11.0)$ & $<0.001$ \\
\hline $\operatorname{BMI}\left(\mathrm{kg} / \mathrm{m}^{2}\right)$ & $27.07 \pm 2.93$ & $27.39 \pm 2.93$ & $26.06 \pm 2.71$ & $<0.001$ \\
\hline Systolic blood pressure (mmHg) & $133.26 \pm 10.90$ & $133.52 \pm 11.29$ & $132.46 \pm 9.57$ & 0.131 \\
\hline Diastolic blood pressure ( $\mathrm{mmHg}$ ) & $81.58 \pm 9.43$ & $81.43 \pm 9.68$ & $82.05 \pm 8.63$ & 0.314 \\
\hline Hypertension, $\mathrm{BP} \geq 140 / 90(\%)$ & $507(45.7)$ & $379(45.3)$ & $128(46.9)$ & 0.644 \\
\hline Fasting plasma glucose $(\mathrm{mg} / \mathrm{dL})$ & $163.68 \pm 27.50$ & $167.72 \pm 28.05$ & $151.29 \pm 21.49$ & $<0.001$ \\
\hline HbA1c (\%) & $8.37 \pm 0.94$ & $8.63 \pm 0.89$ & $7.56 \pm 0.53$ & $<0.001$ \\
\hline Total cholesterol (mg/dL) & $185.24 \pm 46.96$ & $188.48 \pm 49.0$ & $175.29 \pm 38.47$ & $<0.001$ \\
\hline Triglycerides (mg/dL) & $119.07 \pm 62.98$ & $119.47 \pm 63.99$ & $117.84 \pm 59.85$ & 0.702 \\
\hline LDL cholesterol $(\mathbf{m g} / \mathbf{d L})$ & $119.44 \pm 44.34$ & $122.28 \pm 46.41$ & $110.75 \pm 35.98$ & $<0.001$ \\
\hline HDL cholesterol (mg/dL) & $45.05 \pm 10.08$ & $45.67 \pm 10.11$ & $43.13 \pm 9.74$ & $<0.001$ \\
\hline $\operatorname{VLDL}(\mathbf{m g} / \mathbf{d L})$ & $23.68 \pm 12.69$ & $23.81 \pm 12.94$ & $23.30 \pm 11.91$ & 0.559 \\
\hline Blood urea nitrogen $(\mathrm{mg} / \mathrm{dL})$ & $14.62 \pm 3.51$ & $14.60 \pm 3.55$ & $14.70 \pm 3.40$ & 0.698 \\
\hline Creatinine $(\mathrm{mg} / \mathrm{dL})$ & $1.11 \pm 0.17$ & $1.11 \pm 0.17$ & $1.10 \pm 0.17$ & 0.625 \\
\hline $\operatorname{ALT}(\mathbf{I U} / \mathbf{L})$ & $36.06 \pm 13.20$ & $38.81 \pm 12.99$ & $27.63 \pm 9.86$ & $<0.001$ \\
\hline $\operatorname{AST}(\mathbf{I U} / \mathbf{L})$ & $32.49 \pm 12.72$ & $35.06 \pm 12.55$ & $24.59 \pm 9.60$ & $<0.001$ \\
\hline Alk.ph (IU/L) & $108.46 \pm 21.59$ & $108.91 \pm 21.43$ & $107.07 \pm 22.05$ & 0.221 \\
\hline Vit-D (ng/mL) & $21.67 \pm 12.78$ & $19.71 \pm 11.97$ & $27.68 \pm 13.36$ & $<0.001$ \\
\hline 25-OH vitamin D status & & & & $<0.001$ \\
\hline Vit-D $<20$ (deficiency) & $495(44.6)$ & $410(49.0)$ & $85(31.1)$ & \\
\hline Vit-D: 20 - 30 (insufficiency) & $377(34.0)$ & $294(35.1)$ & $83(30.4)$ & \\
\hline Vit-D $\geq 30$ (normal) & $236(21.3)$ & $131(15.7)$ & $105(38.5)$ & \\
\hline Season of blood sampling & & & & 0.934 \\
\hline Spring & $306(27.6)$ & $232(27.7)$ & $74(27.1)$ & \\
\hline Summer & $257(23.2)$ & $191(22.8)$ & $66(24.2)$ & \\
\hline Autumn & $224(20.2)$ & $172(20.5)$ & $52(19.0)$ & \\
\hline Winter & $321(28.9)$ & $241(28.8)$ & $80(29.3)$ & \\
\hline \multicolumn{5}{|l|}{ Vit-D by season of blood sampling } \\
\hline Spring & $22.99 \pm 14.84$ & $21.01 \pm 13.57$ & $29.19 \pm 16.89$ & $<0.001$ \\
\hline Summer & $21.59 \pm 11.55$ & $19.26 \pm 10.62$ & $28.33 \pm 11.55$ & $<0.001$ \\
\hline Autumn & $21.57 \pm 13.22$ & $19.15 \pm 12.57$ & $29.56 \pm 12.23$ & $<0.001$ \\
\hline Winter & $20.49 \pm 11.20$ & $19.15 \pm 10.83$ & $24.51 \pm 11.37$ & $<0.001$ \\
\hline
\end{tabular}

Abbreviations: AST, aspartate transaminase; ALT, alanine transaminase; Alk.ph, alkaline phosphatase; BMI, body mass index; BP, blood pressure; DM, diabetes mellitus; HbAlc, glycated hemoglobin; HDL, high-density lipoprotein; LDL, low-density lipoprotein; VLDL, very-low-density lipoprotein; OHA, oral hypoglycemic agents. a Values are expressed as No. (\%) or mean \pm SD.

${ }^{\mathrm{b}}$ P-values are obtained using independent $t$-test or Pearson $\chi^{2}$ test.

severity of NAFLD, and its deficiency is associated with the exacerbation of NAFLD.

In patients with NAFLD, insulin sensitivity is reduced in the muscles, fat, and liver (39). Vitamin D increases insulin sensitivity by increasing the number of insulin receptors in myocytes, increasing insulin sensitivity in insulin receptors, and affecting peroxisome proliferator-activated receptor delta (PPAR- $\delta$ ) (40). On the other hand, during oxidative stress, an elevation in reactive oxygen species (ROS) and lipid peroxidation occurs, which ultimately leads to intracellular damage (41). The concentrations of lipid peroxidation biomarkers are correlated with the severity of 

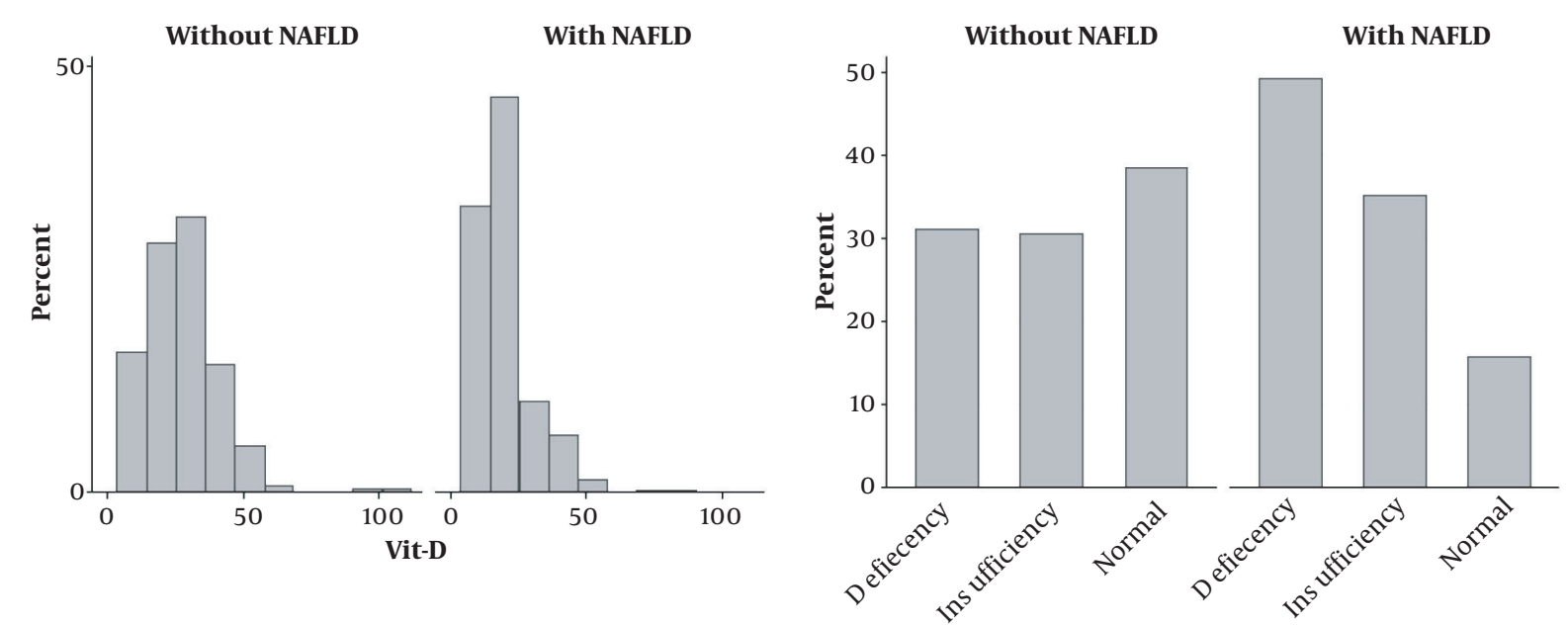

25-OH Vitamin D Status

Figure 1. Vit-D distribution in NAFLD and non-NAFLD patients

\begin{tabular}{|c|c|c|}
\hline Variable & OR $(95 \% \mathrm{CI})$ & P-Value \\
\hline Age $(y)$ & $1.05(1.03$ to 1.06$)$ & $<0.001$ \\
\hline Sex, female & 1.14 (0.856 to 1.51$)$ & 0.375 \\
\hline Diabetes duration & $2.24(2.0$ to 2.51$)$ & $<0.001$ \\
\hline Positive family history of DM & 2.84 (2.13 to 3.77$)$ & $<0.001$ \\
\hline Use of antihypertensive drug & $2.15(1.62$ to 2.84$)$ & $<0.001$ \\
\hline Use of statin & $1.11(0.768$ to 1.62$)$ & 0.570 \\
\hline Use of OHA & $0.463(0.344$ to 0.622$)$ & $<0.001$ \\
\hline Use of insulin & $5.38(3.59$ to 8.05$)$ & $<0.001$ \\
\hline BMI & $1.17(1.12$ to 1.23$)$ & $<0.001$ \\
\hline Systolic blood pressure & $1.01(0.996$ to 1.02$)$ & 0.164 \\
\hline Diastolic blood pressure & $0.993(0.979$ to 1.01$)$ & 0.342 \\
\hline Hypertension, BP $\geq 140 / 90$ & $0.937(0.713$ to 1.23$)$ & 0.644 \\
\hline Fasting plasma glucose & $1.02(1.02$ to 1.03$)$ & $<0.001$ \\
\hline HbA1c & $8.51(6.40$ to 11.33$)$ & $<0.001$ \\
\hline Total cholesterol & $1.01(1.0$ to 1.01$)$ & $<0.001$ \\
\hline Triglycerides & $1.0(0.998$ to 1.0$)$ & 0.711 \\
\hline LDL cholesterol & $1.01(1.0$ to 1.01$)$ & $<0.001$ \\
\hline HDL cholesterol & $1.03(1.01$ to 1.04$)$ & $<0.001$ \\
\hline VLDL & $1.0(0.992$ to 1.01$)$ & 0.559 \\
\hline Blood urea nitrogen & $0.992(0.954$ to 1.03$)$ & 0.698 \\
\hline Creatinine & $1.22(0.553$ to 2.68$)$ & 0.625 \\
\hline ALT & $1.11(1.09$ to 1.12$)$ & $<0.001$ \\
\hline AST & $1.10(1.08$ to 1.12$)$ & $<0.001$ \\
\hline ALK.ph & $1.0(0.998$ to 1.01$)$ & 0.221 \\
\hline Serum vitamin-D level & $0.953(0.942$ to 0.964$)$ & $<0.001$ \\
\hline Vitamin D deficiency vs. normal & $3.87(2.73$ to 5.47$)$ & $<0.001$ \\
\hline Vitamin D insufficiency vs. normal & $2.83(1.99$ to 4.03$)$ & $<0.001$ \\
\hline
\end{tabular}




\begin{tabular}{|c|c|c|}
\hline Variables & OR $(95 \% \mathrm{CI})$ & P-Value \\
\hline \multicolumn{3}{|c|}{ Model 1: Step 1 of Backward Stepwise } \\
\hline Age (y) & 1.07 (1.03 to 1.11$)$ & $<0.001$ \\
\hline Diabetes duration & $2.91(2.35$ to 3.61$)$ & $<0.001$ \\
\hline Positive family history of DM & $1.96(1.04$ to 3.69$)$ & 0.038 \\
\hline Use of antihypertensive drug & 9.15 (4.44 to 18.86$)$ & $<0.001$ \\
\hline Use of insulin & 20.18 (8.74 to 46.61$)$ & $<0.001$ \\
\hline BMI & $1.30(1.15$ to 1.46$)$ & $<0.001$ \\
\hline HbA1c & 11.79 (6.64 to 20.92$)$ & $<0.001$ \\
\hline Total cholesterol & $1.0(0.986$ to 1.02$)$ & 0.724 \\
\hline LDL cholesterol & $1.01(0.988$ to 1.03$)$ & 0.521 \\
\hline HDL cholesterol & 0.998 (0.996 to 1.03$)$ & 0.911 \\
\hline ALT & $1.14(1.05$ to 1.23$)$ & 0.001 \\
\hline AST & 0.990 (0.916 to 1.07$)$ & 0.792 \\
\hline Vitamin D deficiency vs. normal & $3.12(1.36$ to 7.15$)$ & 0.011 \\
\hline Vitamin D insufficiency vs. normal & $2.82(1.26$ to 6.27$)$ & 0.007 \\
\hline \multicolumn{3}{|c|}{ Model 2: Final Step of Backward Stepwise } \\
\hline Age & 1.07 (1.03 to 1.11$)$ & $<0.001$ \\
\hline Diabetes duration & $2.92(2.36$ to 3.61$)$ & $<0.001$ \\
\hline Positive family history of DM & $1.96(1.05$ to 3.69$)$ & 0.036 \\
\hline Use of insulin & 20.30 (8.80 to 46.82$)$ & $<0.001$ \\
\hline BMI & $1.30(1.15$ to 1.46$)$ & $<0.001$ \\
\hline HbAic & 11.76 (6.64 to 20.83$)$ & $<0.001$ \\
\hline LDL cholesterol & $1.01(1.0$ to 1.02$)$ & 0.029 \\
\hline ALT & $1.13(1.09$ to 1.17$)$ & $<0.001$ \\
\hline Vitamin D deficiency vs normal & $3.15(1.38$ to 7.19$)$ & 0.011 \\
\hline Vitamin D insufficiency vs normal & $2.82(1.27$ to 6.28$)$ & 0.006 \\
\hline
\end{tabular}

liver disease (42). According to previous research, vitamin D deficiency increases the concentration of oxidative stress biomarkers, and vitamin D intake reduces the concentration of these biomarkers (43). Also, abnormal lipid metabolism results in fat accumulation in the liver, which in turn increases the production of various adipokines, inflammation, and oxidative stress, all of which play an important role in the NAFLD pathogenesis (44).

This research had some limitations. First, it was a crosssectional research, in which a cause-and-effect relationship could not be indicated between vitamin $D$ deficiency and NAFLD. Second, no liver biopsy was performed in this study. Generally, liver biopsy is the gold standard technique to diagnose NAFLD and differentiate it from NASH. However, considering the aggressiveness of liver biopsy, ultrasound has been used to diagnose NAFLD in previous studies. Ultrasound sensitivity for the diagnosis of NAFLD ranges from 60 to 94\%, depending on the severity of steatosis.

On the other hand, since ultrasound is an operatordependent method, all liver ultrasounds were performed by an experienced radiologist in this study, which is one of its main strengths. Also, elimination of other reasons for chronic liver disease and relatively acceptable sample size are other strengths of this study.

In summary, vitamin D status is associated with the presence of NAFLD in T2DM patients. However, large-scale prospective studies are needed to demonstrate this association and suggest vitamin D deficiency as a risk factor for NAFLD in diabetic patients. Further investigation is warranted to examine the effect of vitamin $D$ supplementation on liver steatosis status in these cases.

\section{Acknowledgments}

The authors wish to thank all the participants.

\section{Footnotes}

Authors' Contribution: Vahid Sheikhi, designed the study and wrote the paper; Zahra Heidari, designed the 
study, performed bioinformatics analyses, and co-wrote the paper.

Conflict of Interests: The authors declared no conflicts of potential interest in conducting this research and its publication.

Data Reproducibility: The data presented in this study are openly available in one of the repositories or will be available on request from the corresponding author by this journal representative at any time during submission or after publication. Otherwise, all consequences of possible withdrawal or future retraction will be with the corresponding author.

Ethical Approval: All procedures performed in this study were in accordance with the ethical standards of the institutional and/or national research committee, as well as the 1964 Helsinki declaration and its later amendments. The Ethics Committee for Human Studies at Zahedan University of Medical Sciences approved the study protocol.

Funding/Support: This study was funded by Zahedan University of Medical Sciences (grant number: 1805003).

Informed Consent: Informed consent was obtained from all participants.

\section{References}

1. Zhu JZ, Hollis-Hansen K, Wan XY, Fei SJ, Pang XL, Meng FD, et al. Clinical guidelines of non-alcoholic fatty liver disease: A systematic review. World J Gastroenterol. 2016;22(36):8226-33. doi: 10.3748/wjg.v22.i36.8226. [PubMed: 27688665]. [PubMed Central: PMC5037092]

2. Starley BQ, Calcagno CJ, Harrison SA. Nonalcoholic fatty liver disease and hepatocellular carcinoma: A weighty connection. Hepatology. 2010;51(5):1820-32. doi: 10.1002/hep.23594. [PubMed: 20432259].

3. Ray K. NAFLD-the next global epidemic. Nat Rev Gastroenterol Hepatol. 2013;10(11):621. doi: 10.1038/nrgastro.2013.197. [PubMed: 24185985].

4. McPherson S, Hardy T, Henderson E, Burt AD, Day CP, Anstee QM. Evidence of NAFLD progression from steatosis to fibrosingsteatohepatitis using paired biopsies: Implications for prognosis and clinical management. J Hepatol. 2015;62(5):1148-55. doi: 10.1016/j.jhep.2014.11.034. [PubMed: 25477264].

5. Clark JM, Diehl AM. Nonalcoholic fatty liver disease: An underrecognized cause of cryptogenic cirrhosis. JAMA. 2003;289(22):3000-4. doi: 10.1001/jama.289.22.3000. [PubMed: 12799409].

6. Samji NS, Verma R, Satapathy SK. Magnitude of Nonalcoholic Fatty Liver Disease: Western Perspective. J Clin Exp Hepatol. 2019;9(4):497505. doi: 10.1016/j.jceh.2019.05.001. [PubMed:31516266]. [PubMed Central: PMC6728535].

7. Andronescu CI, Purcarea MR, Babes PA. Nonalcoholic fatty liver disease: epidemiology, pathogenesis and therapeutic implications. J Med Life. 2018;11(1):20-3. [PubMed: 29696060]. [PubMed Central: PMC5909941].

8. Perumpail BJ, Khan MA, Yoo ER, Cholankeril G, Kim D, Ahmed A. Clinical epidemiology and disease burden of nonalcoholic fatty liver disease. World J Gastroenterol. 2017;23(47):8263-76. doi: 10.3748/wjg.v23.i47.8263. [PubMed: 29307986]. [PubMed Central: PMC5743497].

9. Iqbal U, Perumpail BJ, Akhtar D, Kim D, Ahmed A. The Epidemiology, Risk Profiling and Diagnostic Challenges of Nonalcoholic Fatty Liver Disease. Medicines (Basel). 2019;6(1). doi: 10.3390/medicines6010041. [PubMed: 30889791]. [PubMed Central: PMC6473603].
10. Duseja A, Dhiman RK, Premkumar M. Nonalcoholic Fatty Liver Disease: Lessons Learnt in the Last Five Years. J Clin Exp Hepatol. 2021;11(2):159-62. doi: 10.1016/j.jceh.2020.07.008. [PubMed: 33746439]. [PubMed Central: PMC7953007].

11. Saeedi P, Petersohn I, Salpea P, Malanda B, Karuranga S, Unwin N, et al. Global and regional diabetes prevalence estimates for 2019 and projections for 2030 and 2045: Results from the International Diabetes Federation Diabetes Atlas, 9(th) edition. Diabetes Res Clin Pract. 2019;157:107843. doi: 10.1016/j.diabres.2019.107843. [PubMed: 31518657].

12. Doycheva I, Zhang T, Amjad W, Thuluvath PJ. Diabetes and Hepatocellular Carcinoma: Incidence Trends and Impact of Liver Disease Etiology. J Clin Exp Hepatol. 2020;10(4):296-303. doi: 10.1016/j.jceh.2019.11.004. [PubMed: 32655232]. [PubMed Central: PMC7335702].

13. Sayki Arslan M, Turhan S, Dincer I, Mizrak D, Corapcioglu D, Idilman R. A potential link between endothelial function, cardiovascular risk, and metabolic syndrome in patients with Non-alcoholic fatty liver disease. Diabetol Metab Syndr. 2014;6:109. doi: 10.1186/1758-5996-6-109. [PubMed: 25960770]. [PubMed Central: PMC4424578].

14. Wang XJ, Malhi H. Nonalcoholic Fatty Liver Disease. Ann Intern Med. 2018;169(9):ITC65-80. doi: 10.7326/AITC201811060. [PubMed: 30398639].

15. Chen LW, Chien CY, Hsieh CW, Chang LC, Huang MH, Huang WY, et al. The Associations Between Helicobacter pylori Infection, Serum Vitamin D, and Metabolic Syndrome: A Community-Based Study. Medicine (Baltimore). 2016;95(18). e3616. doi: 10.1097/MD.0000000000003616. [PubMed: 27149497]. [PubMed Central: PMC4863814].

16. Apostolakis M, Armeni E, Bakas P, Lambrinoudaki I. Vitamin D and cardiovascular disease. Maturitas. 2018;115:1-22. doi: 10.1016/j.maturitas.2018.05.010. [PubMed: 30049340].

17. Feldstein AE, Wieckowska A, Lopez AR, Liu YC, Zein NN, McCullough AJ. Cytokeratin-18 fragment levels as noninvasive biomarkers for nonalcoholic steatohepatitis: A multicenter validation study. Hepatology. 2009;50(4):1072-8. doi: 10.1002/hep.23050. [PubMed: 19585618]. [PubMed Central: PMC2757511].

18. Grammatiki M, Rapti E, Karras S, Ajjan RA, Kotsa K. Vitamin D and diabetes mellitus: Causal or casual association? Rev Endocr Metab Disord. 2017;18(2):227-41. doi: 10.1007/s11154-016-9403-y. [PubMed: 28062940].

19. Chung GE, Kim D, Kwak MS, Yang JI, Yim JY, Lim SH, et al. The serum vitamin D level is inversely correlated with nonalcoholic fatty liver disease. Clin Mol Hepatol. 2016;22(1):146-51. doi: 10.3350/cmh.2016.22.1.146. [PubMed: 27044765]. [PubMed Central: PMC4825160].

20. Nelson JE, Roth CL, Wilson LA, Yates KP, Aouizerat B, MorganStevenson V, et al. Vitamin D Deficiency Is Associated With Increased Risk of Non-alcoholic Steatohepatitis in Adults With Non-alcoholic Fatty Liver Disease: Possible Role for MAPK and NF-kappaB? Am J Gastroenterol. 2016;111(6):852-63. doi: 10.1038/ajg.2016.51. [PubMed: 27002799]. [PubMed Central: PMC5361650].

21. Dasarathy J, Periyalwar P, Allampati S, Bhinder V, Hawkins C, Brandt P, et al. Hypovitaminosis $\mathrm{D}$ is associated with increased whole body fat mass and greater severity of non-alcoholic fatty liver disease. Liver Int. 2014;34(6):e118-27. doi: 10.1111/liv.12312. [PubMed: 24118743]. [PubMed Central: PMC4012003].

22. Targher G, Scorletti E, Mantovani A, Byrne CD. Nonalcoholic fatty liver disease and reduced serum vitamin $\mathrm{D}(3)$ levels. Metab Syndr Relat Disord. 2013;11(4):217-28. doi: 10.1089/met.2013.0044. [PubMed: 23745619].

23. Patel YA, Henao R, Moylan CA, Guy CD, Piercy DL, Diehl AM, et al. Vitamin D is Not Associated With Severity in NAFLD: Results of a Paired Clinical and Gene Expression Profile Analysis. Am J Gastroenterol. 2016;111(11):1591-8. doi: 10.1038/ajg.2016.406. [PubMed: 27644736]. [PubMed Central: PMC5331905].

24. Bril F, Maximos M, Portillo-Sanchez P, Biernacki D, Lomonaco R, Subbarayan S, et al. Relationship of vitamin D with insulin resistance and disease severity in non-alcoholic steatohepatitis.J Hepatol. 2015;62(2):405-11. doi:10.1016/j.jhep.2014.08.040. [PubMed: 25195551]. 
25. Tchelepi H, Ralls PW, Radin R, Grant E. Sonography of diffuse liver disease. J Ultrasound Med. 2002;21(9):1023-32. quiz 1033-4. doi: 10.7863/jum.2002.21.9.1023. [PubMed: 12216750].

26. Chalasani N, Younossi Z, Lavine JE, Diehl AM, Brunt EM, Cusi K, et al. The diagnosis and management of non-alcoholic fatty liver disease: Practice guideline by the American Association for the Study of Liver Diseases, American College of Gastroenterology, and the American Gastroenterological Association. Am J Gastroenterol. 2012;107(6):81126. doi: 10.1038/ajg.2012.128. [PubMed: 22641309].

27. Holick MF. The vitamin D deficiency pandemic: Approaches for diagnosis, treatment and prevention. Rev Endocr Metab Disord. 2017;18(2):153-65. doi: 10.1007/s11154-017-9424-1. [PubMed: 28516265].

28. Wang JM, Ye SD, Li SM, Hu W. Correlations of 25(OH)D level with blood lipid, inflammatory factors and vascular endothelial function in diabetic patients. Eur Rev Med Pharmacol Sci. 2018;22(3):731-5. doi: 10.26355/eurrev_201802_14303. [PubMed: 29461603].

29. Hosny SS, Ali HM, Mohammed WA, El Ghannam MH. Study of relationship between total vitamin D level and NAFLD in a sample of Egyptian patients with and without T2DM. Diabetes Metab Syndr. 2019;13(3):1769-71. doi:10.1016/j.dsx.2019.04.002. [PubMed: 31235092].

30. Rhee EJ, Kim MK, Park SE, Park CY, Baek KH, Lee WY, et al. High serum vitamin $\mathrm{D}$ levels reduce the risk for nonalcoholic fatty liver disease in healthy men independent of metabolic syndrome. Endocr J. 2013;60(6):743-52. doi: 10.1507/endocrj.ej12-0387. [PubMed: 23411507].

31. Targher G, Bertolini L, Scala L, Cigolini M, Zenari L, Falezza G, et al. Associations between serum 25-hydroxyvitamin D3 concentrations and liver histology in patients with non-alcoholic fatty liver disease. Nutr Metab Cardiovasc Dis. 2007;17(7):517-24. doi: 10.1016/j.numecd.2006.04.002. [PubMed: 16928437].

32. Li L, Zhang L, Pan S, Wu X, Yin X. No significant association between vitamin $\mathrm{D}$ and nonalcoholic fatty liver disease in a Chinese population. Dig Dis Sci. 2013;58(8):2376-82. doi: 10.1007/s10620-013-2658-1. [PubMed: 23589141].

33. Hong HC, Lee JS, Choi HY, Yang SJ, Yoo HJ, Seo JA, et al. Liver enzymes and vitamin D levels in metabolically healthy but obese individuals: Korean National Health and Nutrition Examination Survey. Metabolism. 2013;62(9):1305-12. doi: 10.1016/j.metabol.2013.04.002. [PubMed: 23643404].

34. Grunhage F, Hochrath K, Krawczyk M, Hoblinger A, ObermayerPietsch B, Geisel J, et al. Common genetic variation in vitamin D metabolism is associated with liver stiffness. Hepatology. 2012;56(5):1883-91. doi: 10.1002/hep.25830. [PubMed: 22576297].

35. Eliades M, Spyrou E. Vitamin D: A new player in non-alcoholic fatty liver disease? World J Gastroenterol. 2015;21(6):1718-27. doi: 10.3748/wjg.v21.i6.1718. [PubMed: 25684936]. [PubMed Central: PMC4323447].

36. Seo YY, Cho YK, Bae JC, Seo MH, Park SE, Rhee EJ, et al. Tumor Necrosis Factor-alpha as a Predictor for the Development of Nonalcoholic Fatty Liver Disease: A 4-Year Follow-Up Study. Endocrinol Metab (Seoul). 2013;28(1):41-5. doi: 10.3803/EnM.2013.28.1.41. [PubMed: 24396649]. [PubMed Central: PMC3811803].

37. Manco M, Marcellini M, Giannone G, Nobili V. Correlation of serum TNF-alpha levels and histologic liver injury scores in pediatric nonalcoholic fatty liver disease. Am J Clin Pathol. 2007;127(6):954-60. doi: 10.1309/6VJ4DWGYDUOXYJ8Q. [PubMed: 17509993].

38. Karkeni E, Bonnet L, Marcotorchino J, Tourniaire F, Astier J, Ye J, et al. Vitamin D limits inflammation-linked microRNA expression in adipocytes in vitro and in vivo: A new mechanism for the regulation of inflammation by vitamin D. Epigenetics. 2018;13(2):156-62. doi: 10.1080/15592294.2016.1276681. [PubMed: 28055298]. [PubMed Central: PMC5873365].

39. Lomonaco R, Ortiz-Lopez C, Orsak B, Webb A, Hardies J, Darland C, et al. Effect of adipose tissue insulin resistance on metabolic parameters and liver histology in obese patients with nonalcoholic fatty liver disease. Hepatology.2012;55(5):1389-97. doi:10.1002/hep.25539. [PubMed: 22183689].

40. Pinelli NR, Jaber LA, Brown MB, Herman WH. Serum 25-hydroxy vitamin $\mathrm{d}$ and insulin resistance, metabolic syndrome, and glucose intolerance among Arab Americans. Diabetes Care. 2010;33(6):13735. doi: 10.2337/dc09-2199. [PubMed: 20332348]. [PubMed Central: PMC2875457].

41. Spahis S, Delvin E, Borys JM, Levy E. Oxidative Stress as a Critical Factor in Nonalcoholic Fatty Liver Disease Pathogenesis. Antioxid Redox Signal. 2017;26(10):519-41. doi: 10.1089/ars.2016.6776. [PubMed: 27452109].

42. Liu S, Shi W, Li G, Jin B, Chen Y, Hu H, et al. Plasma reactive carbonyl species levels and risk of non-alcoholic fatty liver disease. J Gastroenterol Hepatol. 2011;26(6):1010-5. doi: 10.1111/j.1440-1746.2011.06672.x. [PubMed: 21265881].

43. Tamadon MR, Soleimani A, Keneshlou F, Mojarrad MZ, Bahmani F, Naseri A, et al. Clinical Trial on the Effects of Vitamin D Supplementation on Metabolic Profiles in Diabetic Hemodialysis. Horm Metab Res. 2018;50(1):50-5. doi: 10.1055/s-0043-119221. [PubMed: 28958110].

44. Polyzos SA, Kountouras J, Mantzoros CS. Adipokines in nonalcoholic fatty liver disease. Metabolism. 2016;65(8):1062-79. doi: 10.1016/j.metabol.2015.11.006. [PubMed: 26725002]. 